\title{
Uso de Paralelismo em uma Aplicação de Meio Poroso
}

\author{
Ronaldo Canofre M. dos Santos, Mauricio Martinuzzi Fiorenza, \\ Daniel Chaves Temp, Pablo Brauner Viegas, Claudio Schepke \\ ${ }^{1}$ Programa de Pós-Graduação em Engenharia de Software (PPGES) \\ Universidade Federal do Pampa (UNIPAMPA) \\ Alegrete - RS - Brasil \\ \{canofresantos, mauriciofiorenza, claudioschepke\} @unipampa.edu.br \\ danieltemp, pabloviegas\}.aluno@unipampa.edu.br

\begin{abstract}
Resumo. O aumento de desempenho dos processadores atuais, deve-se ao número cada vez maior de cores. Este trabalho apresenta melhorias aplicadas ao código de um projeto denominado Poros, utilizando o paralelismo através da biblioteca OpenMP. Dessa forma, com a análise da execução sequencial em comparação com as execuções paralelas, foi possível verificar uma melhoria de cerca de $23 \%$ no tempo de execução em uma arquitetura multicore.
\end{abstract}

\section{Introdução}

O particionamento de um problema visando a sua execução de forma mais otimizada, não se restringe somente a problemas computacionais, sendo facilmente aplicável a problemas práticos das mais diversas áreas. Nesta linha, é possível buscar a utilização de paralelismo aplicado a problemas já conhecidos, como por exemplo, a adaptação de soluções aplicadas a dinâmica de fluidos, para uma separação de fluxo de grãos [de Oliveira 2020].

Atualmente, o trabalho em desenvolvimento, denominado projeto Poros, realiza esta resolução de forma sequencial, utilizando como base as equações de Navier-Stokes [Constantin and Foias 1988]. No entanto, o desempenho obtido fica aquém das necessidades de tempo e poder de processamento existentes, sendo desejada a otimização da execução na busca por ganhos de eficiência na resolução do problema. Dessa forma, este trabalho apresenta melhorias aplicadas ao código, com a utilização de paralelismo através da biblioteca OpenMP [Chandra et al. 2001].

No decorrer do trabalho, discutimos as alterações/paralelizações realizadas na Seção 2, os resultados obtidos na Seção 3 e as considerações finais do trabalho na Seção 4.

\section{Implementação}

A implementação realizada iniciou-se com a adaptação do código fonte para utilização do OpenMP, incluindo também como opções de compilação, as flags “-O3” para otimização e "-mp" para interpretar as diretivas e pragmas de programação paralela de memória compartilhada, tendo sido utilizado o compilador "pgf90" do pacote Nvidia HPC SDK ${ }^{1}$.

A otimização do código foi realizada sobre o arquivo que reúne as sub-rotinas chamadas durante a execução, sendo concentrada a aplicação de paralelização nos principais laços existentes, observando-se o controle de compartilhamento das variáveis, bem como a dependência temporal das mesmas. Já as execuções foram realizadas como sendo sempre uma nova instância, buscando manter constante o número de iterações.

\footnotetext{
1https://www.pgroup.com/index.htm
} 


\section{Resultados}

Para validação e coleta de resultados, realizou-se a execução do código sequencial (com uma thread) em comparação com execuções paralelas com 2, 3, 4, 5 e 6 threads, obtendo como resultado a média simples de três execuções para cada instância analisada. Tais execuções foram realizadas em um host físico executando Ubuntu Desktop 20.04, equipado com processador Intel(R) Xeon(R) CPU E5-2609 com 1.90GHz, 16 GB de memória e HD $140 \mathrm{~GB}$, em um ambiente de ensino não controlado. Para coleta do tempo total de execução foi utilizado o comando time $e^{2}$. A Tabela 1 mostra os valores temporais coletados em cada execução, bem como a médias obtidas para cada operação.

Tabela 1. Comparativo de execuções sequencial e paralelas

\begin{tabular}{|l|c|c|c|c|c|c|}
\hline & $\mathbf{1}$ Thread & $\mathbf{2}$ Threads & 3 Threads & 4 Threads & $\mathbf{5}$ Threads & 6 Threads \\
\hline Execução 1 & $78,510 \mathrm{~s}$ & $60,486 \mathrm{~s}$ & $59,445 \mathrm{~s}$ & $55,640 \mathrm{~s}$ & $52,710 \mathrm{~s}$ & $50,090 \mathrm{~s}$ \\
Execução 2 & $78,382 \mathrm{~s}$ & $60,713 \mathrm{~s}$ & $58,621 \mathrm{~s}$ & $55,315 \mathrm{~s}$ & $52,808 \mathrm{~s}$ & $50,140 \mathrm{~s}$ \\
Execução 3 & $78,248 \mathrm{~s}$ & $60,953 \mathrm{~s}$ & $58,247 \mathrm{~s}$ & $55,694 \mathrm{~s}$ & $52,498 \mathrm{~s}$ & $50,296 \mathrm{~s}$ \\
\hline Média & $\mathbf{7 8 , 3 8 0 s}$ & $\mathbf{6 0 , 7 1 7 s}$ & $\mathbf{5 8 , 7 7 1 s}$ & $\mathbf{5 5 , 5 5 0 s}$ & $\mathbf{5 2 , 6 7 2 s}$ & $\mathbf{5 0 , 1 7 5 s}$ \\
\hline
\end{tabular}

Os resultados demonstram um ganho de $23 \%$ no tempo de execução, comparando a execução sequencial com a paralela com apenas 2 threads. A melhora no desempenho se confirma conforme são aumentadas os números de processadores, chegando ao máximo de $36 \%$ de melhoria do desempenho com 6 threads paralelas.

\section{Conclusão}

Analisando as execuções realizadas após a aplicação das otimizações com paralelismo via OpenMP, fica visível uma considerável melhoria no tempo de execução, demonstrando que o objetivo foi alcançado, apresentando como resultando uma aplicação com efetiva otimização.

Como trabalhos futuros, pretende-se buscar alternativas de codificação que permitam alcançar resultados melhores dos que os já obtidos, através de técnicas de execução em GPU como OpenACC ou CUDA [Hoshino et al. 2013].

\section{Referências}

Chandra, R., Dagum, L., Kohr, D., Menon, R., Maydan, D., and McDonald, J. (2001). Parallel programming in OpenMP. Morgan kaufmann.

Constantin, P. and Foias, C. (1988). Navier-Stokes Equations. University of Chicago Press.

de Oliveira, D. P. (2020). Fluid Flow Through Porous Media With The One Domain Approach: A Simple Model For Grains Drying. Master's thesis, Universidade Federal do Pampa, Alegrete.

Hoshino, T., Maruyama, N., Matsuoka, S., and Takaki, R. (2013). CUDA vs OpenACC: Performance case studies with kernel benchmarks and a memory-bound CFD application. In 2013 13th IEEE/ACM International Symposium on Cluster, Cloud, and Grid Computing, pages 136-143. IEEE.

\footnotetext{
${ }^{2}$ https://man7.org/linux/man-pages/man1/time.1.html
} 